Aus der Abteilung für Gynäkologie und Geburtshilfe des Landeskrankenhauses Mödling

\section{Eingeladener Kommentar zu: „Die dorsopo- steriore extraperitoneale Pelviskopie (DEP) - eine Präparation an der Leiche}

\section{P. Riss}

In den letzten 20 Jahren hat die Pelviskopie die gesamte Chirurgie im kleinen Becken revolutioniert. Die Gynäkologie hat dabei sicher eine Vorreiterrolle gespielt, haben sich doch die Genitalorgane im kleinen Becken als Objekt für die pelviskopische Betrachtung und in weiterer Folge zur Operation angeboten. So kam es, daß die Pelviskopie sehr rasch weite Verbreitung zur Diagnose von Adhaesionen im kleinen Becken, von Cysten und Myomen sowie von verschlossenen Eileitern fand. Die ersten Anwendungen der operativen Pelviskopie waren dementsprechend Adhaesiolysen, Tubostomien, Zysten- und Adnexexstirpationen und insbesondere die operative Therapie der Tubargravidität.

Die diagnostische Pelviskopie eröffnete bisher unbekannte Möglichkeiten. Die operative Pelviskopie brachte den großen Vorteil, daß ein Bauchschnitt vermieden wurde. Diese Tatsache allein verkürzte die Aufenthaltesdauer für die Patientinnen, verringerte drastisch die postoperativen Schmerzen und reduzierte die Bildung von postoperativen Adhaesionen. Die Manipulation an den Organen und die chirurgische Technik waren weiterhin , invasiv", die Vermeidung der Laparotomie begünstigte aber die Zuteilung zum Begriff ,,minimal invasive Chirurgie".

Die Entwicklung blieb jedoch keineswegs stehen. Einerseits forcierte die Industrie durch die Bereitstellung von immer ausgeklügelteren Instrumenten den Einsatz der Pelviskopie, andererseits erkannten die chirurgisch tätigen Ärztinnen und Ärzte selbst, daß die operative Pelviskopie völlig neue Perspektiven eröffnete. Der Hauptunterschied zur konventionellen Chirurgie liegt in der Tatsache, daß mit der pelviskopischen Optik bis unmittelbar an das Gewebe herangegangen wird. Die Strukturen der Organe und die einzelnen Gewebsschichten mit den Nerven und Gefäßen stellen sich in einer Unmittelbarkeit und Deutlichkeit dar, wie sich aus einer Entfernung von etwa $40 \mathrm{~cm}$ bei der herkömmlichen Chirurgie nicht vorstellbar ist.

Es erstaunt deshalb nicht, daß die Pelviskopie in der Gynäkologie bald als Zugangsweg zur Kolposuspension (lnkontinenz-

Korrespondenzanschrift: Prof. Dr. P. Riss, Abteilung für Gynäkologie und Geburtshilfe. Landeskrankenhaus, A-2340 Mödling. operation) oder zur pelvinen Lymphonodektomie bei onkologíschen Eingriffen eingesetzt wurde. Die Möglichkeit, die einzelnen Schichten besser darzustellen und mit der pelviskopischen Optik zu erkennen hat auch zu Bemühungen geführt, die verschiedenen Teile des Beckenbodens mit dem Laparoskop zu definieren und ansatzweise neue Therapiekonzepte zu entwikkeln.

Die vorgestellte Technik der dorsoposterioren extraperitonealen Pelivskopie stellt einen solchen Ansatz dar. Die Autoren nennen selbst als eine der Möglichkeiten den Zugang zum oberen Scheidendrittel von hinten. Hier ist an eventuelle Eingriff bei Deszensus und Prolaps von Scheide und Uterus bzw. ganz allgemein bei Beckenbodeninsuffizienz zu denken. Unsere Kenntnisse über die morphologische und insbesondere funktionellen $\mathrm{Zu}$ sammenhänge des Beckenbodens sind jedoch noch immer äußerst begrenzt. Kritisch gesehen, kann man sich die Frage stellen, ob es sich hier nicht um einen Fall von ,,Schwanz wackelt mit Hund" handelt. Bringt die neue Technik eine bessere Kenntnis des Beckenbodens, oder müssen wir nicht uns zuerst um ein besseres Verständnis der muskulären und bindegewebigen Strukturen im kleinen Becken bemịhen, bevor wir extraperitoneale Pelviskopie einsetzen?

Pelviskopie darf nie ..l'art pour l'art" sein. 3 Fragen müssen positiv beantwortet werden, um den Einsatz der oft sehr teuren Pelviskopie zu rechtfertigen:

1. Besserer postoperativer Heilungsverlauf"? Die Pelviskopie muß geringere postoperative Schmerzen, weniger fieberhafte Verläufe und weniger Komplikationen gegenüber den etablierten Methoden aufweisen.

2. Bessere Operationsmöglichkeit? Es genügt nicht, daß Eingriffe auch pelviskopisch durchgeführt werden können. Wirtschaftliche Uberlegungen sowie die Operationsdauer sprechen sehr oft gegen den Einsatz der Pelviskopie. Es müssen deshalb auch für die praktische Durchführung des Eingriffes selbst Vorteile erkennbar sein.

3. Bessere Ergebnisse? Was jedoch wirklich zählt, ist das $\mathbb{E}_{\mathfrak{r}}$ gebnis einer Operation. Dieses darf auf keinen Fall schlechter als bei herkömmlichen Methoden sein. Oft hat man den Eindruck, daß der Einsatz von ,,modernen" Techniken und die ,,minimale Invasivität" als Rechtfertigung für den pelviskopischen Zugang genügen.

Ich bin überzeugt, daß wir erst am Anfang der Entwicklung der Pelviskopie stehen. Die Autoren der vorliegenden Arbeit haben einen neuen und interessanten Zugangsweg aufgezeigt. Bei aller Euphorie dürfen wir aber nie die Selbstkritik und die wissenschaftliche Ehrlichkeit außer acht lassen. Nur dann werden wir erkennen, welche Zugangswege und welche Operationen mittels Pelviskopie einen Benefit für die Patientin und den Patienten bringen.

IMPRESSUM: Verleger: Blackwell Wissenschafts-Verlag GesmbH. - Herausgeber: Blackwell Wissenschafts-Veriag GesmbH., gemeinsam mit der Österreichischen Gesellschaf̂́ für Chirurgie und deren assozilierten Fachcesellschaften. - Eigeniümer: Blackwell Science Lld. Osney Mead, Oxford, GB. - Senior Editors: F. Piza. M. D. Wien. F. Helmer, M. D., Wien. - Editor-in-Chiế: B. Niederle, M. D., Wien. - Co-Edisors: P. Steindorfer, M. D., Graz, L. Ch. Müller, M. D., Innsbruck. - Alle: Zehetnergasse 6,

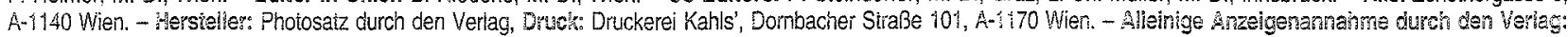
Markus Schulz - Alle: Zehetnergasse 6, A-1140 Wien, Tel +43/1/894 06 90, Fax: +43/1/894 0690 24, E-Mail biack@ via.ä́; Kurfürstendamm 57, D-10707 Berlin, +49/30/32 79 06-0, Fax: +49/30/32 79 06-10. - Abonnementgebühr: Ganzjährig S 2.570,-- Einzelheft S 451,--, alles inklusive Mehwwertsteuer, plus Versandspesen und Manipulation. Für Mitglieder der mitherausgebenden Gesellschaften und für Studierende der Medizin (bei Bezug direkt vom Verlag) emmäBigt sich der Bezugspreis auf jährlich S 1.260,- (zuzüglich Versandspesen und Manipulation).

Die Bezugsdauer veriängert sich um jeweils ein Jahr, wenn nicht spätestens 6 Wochen vor Ablauf des Kalenderjahres gekündigt wird. Diese Zeitschrift ist urheberrechtlich geschützt. Die dadurch begründeten Rechte, insbesondere die der Übersetzung, des Nachdrucks, des Vortrags, der Entnahme van Abbildungen und Tabellen, der Funksendung, der Mikroverfilmung oder Vervielfältigung auf anderen Wegen und der Speicherung in Datenverarbeitungsanlagen, bleiben, auch bei nur auszugsweiser Verwertung, vorbehalten. Eine Vervielfältigung der Zeitschrift oder von Teilen daraus ist auch im Einzelfall nur in den Grenzen der Bestimmungen der einschlägigen gesetzlichen Regelungen zulässig. Sie ist grundsätzlich vergü̈ungspfilichtig. Zuwiderhandlungen unterliegen den Strafbestimmungen der Gesetze.

Die Wiedergabe von Gebrauchsnamen, Handelsnamen, Warenbezeichungen usw. in dieser Zeitschrift berechtigt auch ohne besondere Kennzeichnung nicht zur Annahme, daß solche Namen im Sinne der Warenzeichen- und Markenschutz-Gesetzgebung als frei zu betrachten wären und daher von jedermann benutzt werden dürien.

Produkthaftung: Für Angaben über Dosierungsanweisungen und Applikationsformen kann vom Verlag keine Gewähr übernommen werden. Derartige Angaben müssen vom jeweiligen Anwender im Einzelfall an Hand anderer Literalurstellen aut ihre Richtigkeit überprüft werden. 